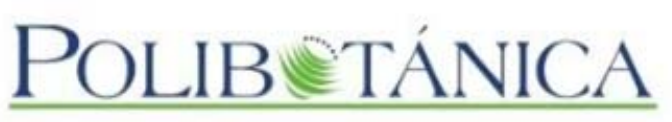

\title{
Polibotánica
}

ISSN electrónico: 2395-9525

polibotanica@gmail.com

Instituto Politécnico Nacional

México

http://www.polibotanica.mx

\section{CAMBIOS BIOQUÍMICOS EN \\ RESPUESTA AL ATAQUE DE ROYA EN PLANTACIONES DE CAFÉ.}

\section{BIOCHEMICAL CHANGES IN RESPONSE TO RUST ATTACK IN COFFEE PLANTATIONS.}

Luján-Hidalgo, M. C., L. A. Jiménez-Aguilar, N. Ruiz-Lau, S. J. Reyes-Zambrano y F. A. Gutiérrez-Miceli.

CAMBIOS BIOQUÍMICOS EN RESPUESTA AL ATAQUE DE ROYA EN PLANTACIONES DE CAFÉ.

BIOCHEMICAL CHANGES IN RESPONSE TO RUST ATTACK IN COFFEE PLANTATIONS.

\section{POLIBETÁNICA \\ Instituto Politécnico Nacional}

Núm. 49: 149-160 México. Enero 2020

DOI: $10.18387 /$ polibotanica.49.10

(c) (i) Este es un artículo de acceso abierto bajo la licencia Creative Commons 4.0 Atribución-No Comercial (CC BY-NC 4.0 Internacional). 


\section{CAMBIOS BIOQUÍMICOS EN RESPUESTA AL ATAQUE DE ROYA EN PLANTACIONES DE CAFÉ.}

\section{BIOCHEMICAL CHANGES IN RESPONSE TO RUST ATTACK IN COFFEE PLANTATIONS.}

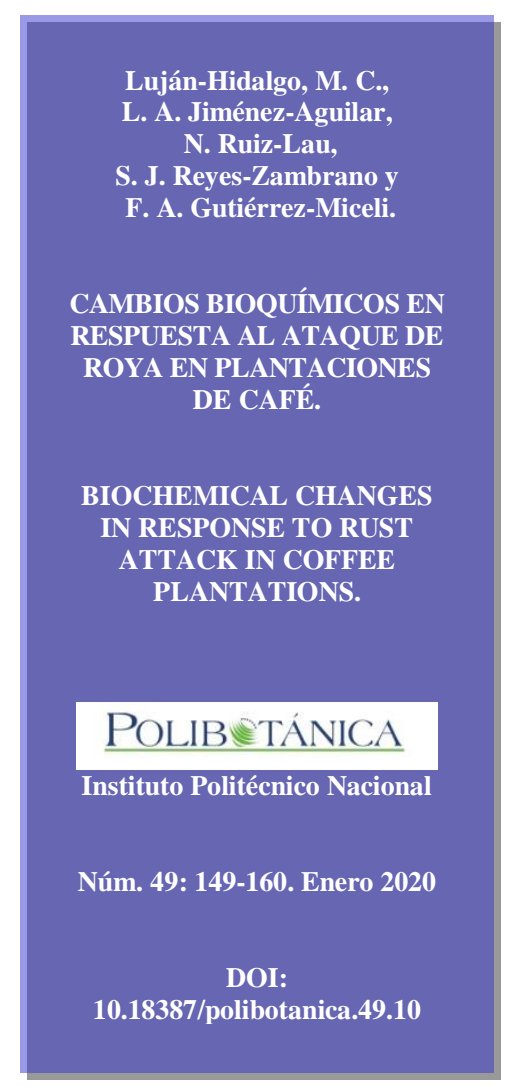

Luján-Hidalgo, M. C.,

. Jiménez-Aguilar,

N. Ruiz-Lau,

S. J. Reyes-Zambrano y

F. A. Gutiérrez-Miceli.

CAMBIOS BIOQUÍMICOS EN

RESPUESTA AL ATAQUE DE

DE CAFÉ.

BIOCHEMICAL CHANGES

N RESPONSE TO RUST

TTACK IN COFFEE

PLANTATIONS.

Instituto Politécnico Nacional

Núm. 49: 149-160. Enero 2020

10.18387/polibotanica.49.10

\begin{abstract}
M. C. Luján-Hidalgo
L. A. Jiménez-Aguilar

N. Ruiz-Lau*

S. J. Reyes-Zambrano

F. A. Gutiérrez-Miceli / fgmiceli@gmail.com

Laboratorio de Biotecnología Vegetal, Instituto Tecnológico de Tuxtla-Gutiérrez, Tuxtla-Gutiérrez, México. Carretera Panamericana km 1080, CP 29050, Chiapas, México.

*Cátedra CONACyT.
\end{abstract}

RESUMEN: Las plantas utilizan diversos mecanismos para defenderse del ataque de hongos patógenos. Algunos de estos mecanismos incluyen el aumento de metabolitos secundarios, antioxidantes, enzimas hidrolíticas y polisacáridos que causan la muerte $o$ reducen la progresión de hongos patógenos. Hemileia vastatrix (roya del café) es un hongo biótrofo que causa graves daños a las plantaciones de café. Ocasionalmente, se pueden observar plantas con ausencia de síntomas. El estudio de estas plantas asintomáticas es de suma importancia para la conservación y mejoramiento del germoplasma resistente a $H$. vastatrix. Para el estudio, aquellas plantas que durante la incidencia de la enfermedad no presentaban síntomas fueron denominadas no infectadas (Ni) y las que si los presentaban como infectadas (i). Se evaluaron dos periodos: antes de la infección por roya y durante ésta, en plantas de Coffea arabica $\mathrm{Ni}$ e i cultivadas en campo bajo un sistema de producción orgánico. Se determinó la actividad enzimática de quitinasa, $\beta-1-3$ glucanasa y peroxidasa; además, de la cuantificación de fenoles totales, ácido clorogénico (3-CQA), ácido criptoclorogénico (4-CQA), ácido neoclorogénico (5-CQA), ácido ferúlico (AF), ácido cafeico (AC), ácido gálico (AG) y cafeína $(\mathrm{Caf})$. No se encontraron diferencias $(\mathrm{p}<0.05)$ en la concentración de 3-CQA y ácido cafeico en las hojas de plantas i y $\mathrm{Ni}$ en los dos periodos evaluados. La concentración de 5-CQA fue 5.3 veces menor en plantas $\mathrm{Ni}$ en comparación con las i durante la infección por roya, sin embargo, este metabolito no se detectó antes de la incidencia de ésta. La concentración de ácido ferúlico fue mayor en las $\mathrm{Ni}$ antes de la infección, mientras que el contenido de ácido gálico en las i. La actividad de las quitinasas fue mayor en las $\mathrm{Ni}$ y esta actividad aumentó durante la infección, la de glucanasas fue mayor en las i y las peroxidasas fueron mayores en las $\mathrm{Ni}$ en los dos periodos. Los resultados sugieren que la resistencia de las plantas de café a la enfermedad por roya está relacionada por la presencia de compuestos fenólicos y proteínas relacionadas con la patogénesis (PR) como la quitinasa, glucanasa y peroxidasa.

Palabras clave: Cafeína; actividad de quitinasa; actividad de glucanasa, peroxidasa.

ABSTRACT: Plants use various mechanisms to defend against pathogenic fungi attack. Some of these mechanisms include the increase of secondary metabolites, antioxidant and hydrolytic enzymes and polysaccharides that cause death or reduce the progression of pathogenic fungi. Hemileia vastatrix (coffee rust) is a biotrofo fungus 
that causes severe damage to coffee plantations. Occasionally, plants with rust absence of symptoms may be observed in coffee plantations. The study of these asymptomatic plants is of paramount importance for conservation and breeding of resistant germplasm to $H$. vastatrix. For the study, plants that did not have symptoms during the incidence of the disease were called uninfected (Ni) and those that had them infected (i). Two periods were evaluated: before and during rust infection, in $\mathrm{Ni}$ and i plants grown in the field under an organic production system. In this study enzymatic activity of quitinase, $\beta-1-3$ glucanase and peroxidase were determined. Additionally, total phenols, chlorogenic (3-CQA), criptochlorogenic (4-CQA), neochlorogenic (5-CQA), pherulic (AF), cafeico (AC), gallic acids and cafeine (Caf) were quantified in infected or non-infected $C$. arabica plants. No differences $(\mathrm{p}<0.05)$ were found in the concentration of 3-CQA and caffeic acid in the $i$ and Ni plants in the two periods evaluated. The concentration of 5-CQA was 5.3 times lower in Ni plants compared to i during rust infection, however, this metabolite was not detected prior to the incidence of rust infection. The concentration of ferulic acid was higher in the $\mathrm{Ni}$ before infection, while the content of gallic acid in the i. Chitinase activity was higher in $\mathrm{Ni}$ and this activity increased during infection, glucanases was higher in $\mathrm{i}$ and peroxidase was higher in $\mathrm{Ni}$ in both periods. The results suggest that the resistance of coffee plants to rust disease is related to the presence of phenolic compounds and pathogenesisrelated proteins (PR) such as chitinases, glucanases and peroxidase activities.

Key words: Caffeine, chitinase activity, glucanase activity, peroxidase.

\section{INTRODUCCIÓN}

El café (Coffea arabica) es una de las plantas más cultivadas y comercializadas en México, siendo Chiapas el principal estado productor de café orgánico (Temis, Lópes \& Sosa, 2011). Uno de los principales problemas en la producción de café es la enfermedad de la roya, la cual puede causar pérdidas de rendimiento de hasta el 50\% (Florez et al., 2017). Actualmente, es el principal problema al que se enfrenta el sector cafetalero en México. El agente causal es Hemileia vastatrix, el cual es un hongo biótrofo que depende completamente de las células vivas de la planta para su reproducción (Florez et al., 2017), por lo que afecta principalmente el follaje de $C$. arabica, provocando una defoliación total de las plantas hasta ocasionar su muerte, originando así, pérdidas de hasta un $40 \%$ de los cultivos (Fernandez et al., 2012). Para el control de éste hongo, se han empleado fungicidas a base de cobre, triazoles y estroburilinas, microorganismos antagónicos, defoliaciones, por mencionar algunos (Fernandez et al., 2012; Haddad, Saraiva, Mizubuti, Romeiro \& Maffia, 2013). Sin embargo, se sabe que las plantas tienen numerosos mecanismos de defensa para contrarrestar los efectos ocasionados por el estrés biótico y abiótico. En el caso del estrés biótico, las plantas activan mecanismos inducidos a través de la respuesta hipersensible, ocasionando la acumulación de metabolitos (ya sea los que se encuentran como componentes estructurales constitutivos como callosa y lignina) y la biosíntesis de fenilpropanoides, así como induciendo la actividad de enzimas antioxidantes (Castillo, Hernández, Gallegos, Rodríguez, \& Aguilar, 2012).

Las estrategias empleadas para controlar el hongo han permitido elucidar algunos mecanismos de defensa que tienen las plantas de café para contrarrestar el agente infeccioso, tales como la expresión genética de enzimas antioxidativas e hidrolíticas y algunas relacionadas a la patogénesis (ejemplo: glucanasa, catalasa, y fenilalanina amonio liasa). Las respuestas defensivas de la planta pueden actuar inhibiendo la expresión de genes del hongo e incluso en la degradación de la pared fúngica, sin embargo, la infección aún no se ha logrado erradicar, y los agricultores tienden a estar en constante vigilancia para mantener el control de la rápida diseminación de la enfermedad (Loureiro, Gil, Maria, Talhinhas, \& Money, 2015).

La enfermedad de la roya del café, causada por el hongo $H$. vastatrix, es una de las principales enfermedades del café en todo el mundo. El uso de cultivares resistentes es considerada una de las estrategias de control más efectiva para esta enfermedad (Florez et al., 2017). En ocasiones, en los cultivos de café infectados con $H$. vastatrix se pueden observar plantas con ausencia de 
síntomas. El estudio de éstas plantas asintomáticas, es de suma importancia y más aun tratándose de conservación y preservación del germoplasma resistente a $H$. vastatrix. De igual manera el desarrollo de las plantas y el rendimiento de la producción de café, son también factores muy importantes para los agricultores (Castillo et al., 2012).

El estudio de plantas asintomáticas ante la incidencia de la roya en cultivos de café orgánico es de vital interés para establecer programas de propagación de germoplasma y de mejoramiento, por lo que el presente trabajo tuvo como objetivo determinar la actividad enzimática de quitinasa, $\beta$-1,3-glucanasa y peroxidasa, cuantificación de fenoles totales, ácido clorogénico, ácido criptoclorogénico, ácido neoclorogénico, ácido ferúlico, ácido cafeico, ácido gálico y cafeína en plantas infectadas (sintomáticas) y no infectadas (asintomáticas) de C. arabica cultivadas en un sistema de producción orgánico, con muestreos antes y durante la incidencia de la infección por roya de manera natural en campo. Se espera relacionar la presencia y/o concentración de compuestos fenólicos y la actividad de proteínas relacionadas con la patogénesis (PR) como las quitinasas, glucanasas y peroxidasas con la resistencia a la enfermedad.

\section{Material y Métodos}

\section{Área de estudio y colecta de muestras}

Se estableció el área de colecta en las plantaciones de café ubicadas en la localidad de Chaná, municipio de Tenejapa, Chiapas (HO-92 $2^{\circ} 9^{\prime} 00.1$; N-16 $53^{\prime} 30.6$; altitud: $1591 \mathrm{~m}$ ) en donde las temperaturas oscilan entre $12^{\circ} \mathrm{C}$ y $26^{\circ} \mathrm{C}$. La incidencia de la enfermedad por roya de manera natural se registra en épocas de lluvias; por lo cual, las colectas se llevaron a cabo en dos temporadas: 1) antes de las lluvias (sin incidencia de roya-antes de la infección (ai)) y 2) después de éstas (con incidencia de roya-durante la infección (di)) en plantas susceptibles y no susceptibles a la enfermedad. Las plantas susceptibles fueron denominadas infectadas (i) y las no susceptibles no infectadas (Ni). Las hojas fueron colectadas al azar a partir de ramas ubicadas en la parte media-superior del tallo (FERTILAB, 2012) y de las mismas plantas para ambas colectas.

\section{Identificación de esporas de $\boldsymbol{H}$. vastatrix}

Se empleó el método reportado por Silva, Nicole, Rijo, Geiger \& Rodrigues (1999). Se aplicó esmalte de uñas transparente sobre la superficie infectada en las hojas, se dejó secar a temperatura ambiente, posteriormente se retiró y se fijaron en portaobjetos. Las muestras se tiñeron con algodón azul de lactofenol, para identificar las esporas del hongo mediante la comparación de las características morfológicas reportadas, como el tamaño, forma y estructura. Se realizaron cortes de las secciones transversales de fragmentos de hojas infectadas y no infectadas hechas con un micrótomo y se tiñó en algodón azul de lactofenol para evaluar la inserción de las urediniosporas del hongo dentro de los tejidos del huésped usando un microscopio de luz de la marca Axiostar plus.

Extracción y determinación de compuestos fenólicos de las hojas de café (Coffea arabica sp.) La extracción de compuestos fenólicos se realizó a partir de hojas deshidratadas y molidas, empleando el método de extracción asistida con ultrasonido reportado por Cuesta-Parra \& Correa-Mahecha (2018) con algunas modificaciones. Se empleó $1 \mathrm{~g}$ de muestra y se adicionaron $10 \mathrm{~mL}$ de una solución de metanol al $80 \%$, el tratamiento con ultrasonido fue por 20 minutos en condiciones de oscuridad; posteriormente el extracto se filtró con papel filtro whatman $\mathrm{N}^{\circ} 2$ realizando lavados con $5 \mathrm{~mL}$ de metanol al $100 \%$ por duplicado. La extracción se repitió dos veces y se recolectó en un matraz balón de $50 \mathrm{~mL}$. El disolvente se rotaevaporó a $40-45^{\circ} \mathrm{C}$ y el agua restante se eliminó por liofilización (LABCONCO FreeZone 4.5 Plus). El extracto se almacenó a $-20{ }^{\circ} \mathrm{C}$ hasta la determinación de fenoles totales y los análisis cromatográficos. 


\section{Cuantificación de fenoles totales}

Para la determinación de fenoles totales (FT) se utilizó el método reportado por Vignoli, Bassoli \& Benassi (2011) con algunas modificaciones. Las muestras liofilizadas se resuspendieron en $3 \mathrm{~mL}$ de metanol al $100 \%$ y se tomaron alícuotas de $100 \mu \mathrm{L}$ del extracto fenólico y se diluyeron con $9 \mathrm{~mL}$ de $\mathrm{H}_{2} \mathrm{O}$ destilada. Se tomaron $20 \mu \mathrm{L}$ de la solución con adición de 1.5 de $\mathrm{mL}$ de agua destilada y $100 \mu \mathrm{L}$ del reactivo Folin and Ciocalteu (SigmaAldrich), se agitó por 5 min y posteriormente se agregaron $300 \mu \mathrm{L}$ de $\mathrm{Na}_{2} \mathrm{CO}_{3}$ al $20 \% \mathrm{p} / \mathrm{v}$, agitando vigorosamente. Se incubó por $90 \mathrm{~min}$ en oscuridad y se medió la absorbancia a 765 $\mathrm{nm}$. El ácido gálico se empleó como estándar para la curva patrón empleando la misma metodología $(0.1-1.0 \mathrm{mg} / \mathrm{mL})$ y agua como blanco. El contenido de fenoles totales del material vegetal se expresó en mg equivalentes de ácido gálico (AG)/gr de muestra seca.

\section{Cuantificación de metabolitos secundarios}

Los metabolitos secundarios de ácido clorogénico (3-CQA), criptoclorogénico (4-CQA), neoclorogénico (5-CQA), ácido ferúlico (AF), ácido cafeico (AC), cafeína (Caf) y ácido gálico (AG) fueron identificados y cuantificados mediante cromatografía líquida de fase reversa (RFHPLC). Aproximadamente $3 \mathrm{mg}$ de cada muestra liofilizada fue resuspendida en $1 \mathrm{~mL}$ de agua tridestilada $\left(\mathrm{H}_{2} \mathrm{Ot}\right)$ y filtrada con una membrana millipore de $0.25 \mu \mathrm{m}$. El equipo que se utilizó fue un HPLC (PerkinElmer Series 200, Shelton, CT, USA), acoplado a un detector DAD. La columna empleada fue una Kromasil 100-5-C18 (4.6x150 mm, $5 \mu \mathrm{m}, 100 \mathrm{~A}$ (SUPELCO, Bellefonte, CA, USA) con un flujo de $0.5 \mathrm{~mL} / \mathrm{min}$. La fase móvil consistió en $95 \%$ de Solvente A: acetonitrilo/ácido fórmico al 0.1\% (80:20 v/v) (Fermont, México; Baker, México) y 5\% de Solvente B: ácido fórmico al 1\% (v/v) en modo isocrático; el análisis se realizó por triplicado a 280 y $320 \mathrm{~nm}$ con un volumen de inyección de $10 \mu \mathrm{L}$ de muestra. Para la elaboración de la curva se utilizaron estándares de 3-CQA, 4-CQA, 5-CQA, AF, AC, Caf y AG. Los tiempos de retención obtenidos para cada compuesto con la curva fueron: 42.67, 44.32, 35.42, 49.15, $43.49,41.88,15.2$ min respectivamente. Los metabolitos cuantificados fueron expresados en sus equivalentes analizados del estándar en $\mathrm{mg} / \mathrm{gr}$ de hoja seca (HS).

\section{Determinación de la actividad enzimática \\ Extracción de proteínas}

Para obtener el extracto total proteico, el tejido foliar fue triturado (300 $\mathrm{mg}$ de materia fresca) en un mortero con nitrógeno líquido. Posteriormente, se le adicionó $1 \mathrm{~mL}$ del amortiguador de extracción [0.1 M Tris- $\mathrm{HCl}(\mathrm{pH} 8.0)]$, ácido ascórbico al $0.1 \%(\mathrm{p} / \mathrm{v})$, glicerina al 10\% (v/v), polivinilpirrolidona al $1 \%(\mathrm{p} / \mathrm{v})$ y $\beta$-mercaptoetanol al $5 \%(\mathrm{p} / \mathrm{v})$ ] y se incubó por $4 \mathrm{~h}$ a $4{ }^{\circ} \mathrm{C}$, por último, las muestras fueron centrifugadas a $15,000 \mathrm{~g}$ por 15 minutos a $4{ }^{\circ} \mathrm{C}$, y almacenadas en un ultracongelador a $-80^{\circ} \mathrm{C}$ hasta su análisis. La concentración de proteína total soluble en los extractos se determinó por el método espectrofotométrico de Bradford de acuerdo a la metodología reportada por Diniz et al. (2019) utilizando albúmina de suero bovino (BSA) como estándar, para la construcción de la curva patrón (10.0-100.0 $\mu \mathrm{g}$ de BSA $/ \mathrm{mL}$ ) (Quick Start ${ }^{\mathrm{TM}}$ Bradford protein assay). La absorbancia se midió a $595 \mathrm{~nm}$ y los resultados se expresaron en $\mu \mathrm{g}$ BSA/gr HS.

\section{Actividad quitinasa}

Se determinó utilizando el método descrito por Honorato, Zambolim, Aucique, Resende \& Rodrigues (2015) con ligeras modificaciones. La reacción se llevó a cabo utilizando $40 \mu \mathrm{L}$ $(0.45 \mu \mathrm{g} / \mu \mathrm{L})$ del extracto total proteico en $940 \mu \mathrm{L}$ de la mezcla de reacción (tampón de acetato de sodio $50 \mathrm{mM}(\mathrm{pH} 5.0)$ ) y $20 \mu \mathrm{L}$ del sustrato p-nitrofenil- $\beta$-DN-N diacetilocitobiosa (SigmaAldrich, São Paulo, Brasil) a una concentración de $2 \mathrm{mg} / \mathrm{mL}$ y se incubó en un baño de agua a $37{ }^{\circ} \mathrm{C}$ durante $2 \mathrm{~h}$. La reacción se detuvo con $1000 \mu \mathrm{L}$ de carbonato de sodio $0.2 \mathrm{M}$. En las muestras control, solo se utilizó carbonato de sodio después de añadir el extracto de enzima a la mezcla de reacción y las muestras se incubaron en un baño de agua a $37{ }^{\circ} \mathrm{C}$ durante $2 \mathrm{~h}$. La absorbancia del producto final liberado por la quitinasa se midió a $410 \mathrm{~nm}$. Se utilizó el coeficiente de extinción molar de $7 \times 10^{4} \mathrm{mM} / \mathrm{cm}$ para calcular la actividad y los resultados fueron expresados en $\mu \mathrm{mol}$ p-nitrofenilo/min*mg de proteína y éstos equivalen a una unidad de 
quitinasa, siendo una unidad la cantidad de enzima que se produce por un $\mu$ mol pnitrofenilo/min, bajo las condiciones antes descritas.

\section{Actividad glucanasa}

Se determinó como describe Dogra \& Sreenivasulu (2015) a partir de $40 \mu \mathrm{L}(0.45 \mu \mathrm{g} / \mu \mathrm{L}) \mathrm{del}$ extracto total proteico y $460 \mu \mathrm{L}$ de la mezcla de reacción (tampón de acetato sódico $100 \mathrm{mM}$ (pH 5.0) y $500 \mu \mathrm{L}$ del sustrato laminarína (4 mg/mL Sigma-Aldrich, São Paulo, Brasil) y se incubó en un baño de agua durante $30 \mathrm{~min}$ a $45{ }^{\circ} \mathrm{C}$. Posteriormente, se determinaron los azúcares reductores con $1000 \mu \mathrm{L}$ de ácido 3,5 dinitrosalicílico, incubando la mezcla en un baño de agua por 15 minutos a $100{ }^{\circ} \mathrm{C}$ y después a $30{ }^{\circ} \mathrm{C}$. El testigo se realizó con el extracto total proteico añadido después de calentar la mezcla a $100{ }^{\circ} \mathrm{C}$. La absorbancia del producto liberado por glucanasa se midió a $540 \mathrm{~nm}$, y la actividad glucanasa se expresó como unidad de actividad (UA)/mg de proteína x min (Ramada et al., 2010).

\section{Actividad peroxidasa}

Se determinó sobre la oxidación del pirogalol de acuerdo con el método de Honorato et al. (2015). Se añadió una mezcla de $590 \mu \mathrm{L}$ de $\mathrm{H}_{2} \mathrm{O}_{2}, 500 \mu \mathrm{L}$ de tampón de fosfato de potasio 100 $\mathrm{mM}$ (pH 6.8), $400 \mu \mathrm{L}$ de pirogalol $100 \mathrm{mM}$ y $400 \mu \mathrm{L}$ de peróxido de hidrógeno $100 \mathrm{mM}$ a 110 $\mu \mathrm{L}(0.45 \mu \mathrm{g} / \mu \mathrm{L})$ del extracto total proteico. La absorbancia se medió con un espectrofotómetro a $420 \mathrm{~nm}$ cada $10 \mathrm{~s}$ durante $1 \mathrm{~min}$ a $25^{\circ} \mathrm{C}$ después de la adición del extracto total proteico. La actividad peroxidasa se calculó mediante el coeficiente de extinción molar de $2.47 / \mathrm{mM} \mathrm{x} \mathrm{cm}$ (Chance \& Maehley, 1955) que se expresó en $\mathrm{mM}$ de purpurogalina/min x mg de proteína. Durante la reacción las muestras se protegieron de la luz para evitar su oxidación.

\section{Diseño experimental y análisis de datos}

Todos los experimentos se realizaron con un diseño completamente al azar con 10 repeticiones y dos tratamientos designados como plantas infectadas y no infectadas con $H$. vastatrix. Una planta corresponde a una unidad observacional. Se utilizaron 20 plantas en cada experimento (10 plantas por cada tratamiento) en dos tiempos de evaluación antes de las lluvias (sin incidencia de roya-antes de la infección) y después de éstas (con incidencia de roya-durante la infección). Los datos de todas las variables se sometieron a un análisis de varianza y las medias de los tratamientos se compararon mediante la prueba de Tukey $(p \leq 05)$ usando el software Statgraphics Centurion XVI.

\section{REsultados}

En la figura 1 se observan los cortes longitudinales en el tejido foliar obtenidos de plantas infectadas con roya. Se visualizaron las estomas y dentro de estos algunos presentaban esporas (fig. 1B); así como, aglomerados de urediniosporas, hifas septadas de germinación e hifas de germinación unidas por diferentes urediniosporas (fig. 1C). Los estomas fueron asimétricos (fig. 1D). La morfología de las urediniosporas mostró una coloración amarillenta en las partes centrales y/o poco traslucidas en otras. También se pudo observar que se mantienen unidas mediante tubos germinativos. En las plantas $\mathrm{Ni}$ no se observaron lesiones en las estomas, tampoco se visualizaron urediniosporas. La peculiaridad del proceso infectivo de las urediniosporas de $H$. vastatrix es a través de los estomas, esta penetración desarrolla haustorios que atacarán principalmente la cavidad subestomática y finalmente lesionándolas. Las plantas no infectadas no presentaron urediniosporas en el proceso de invasión, desarrollo y proceso infectivo. Los daños ocasionados por la roya sobre las hojas manifiestan lesiones redondas de aproximadamente unos $3 \mathrm{~mm}$ en el haz y en el envés, también manifiestan necrosis, con un color amarillo-anaranjado incluso una coloración parda que van desde el centro de la hoja hasta el área perimetral. 

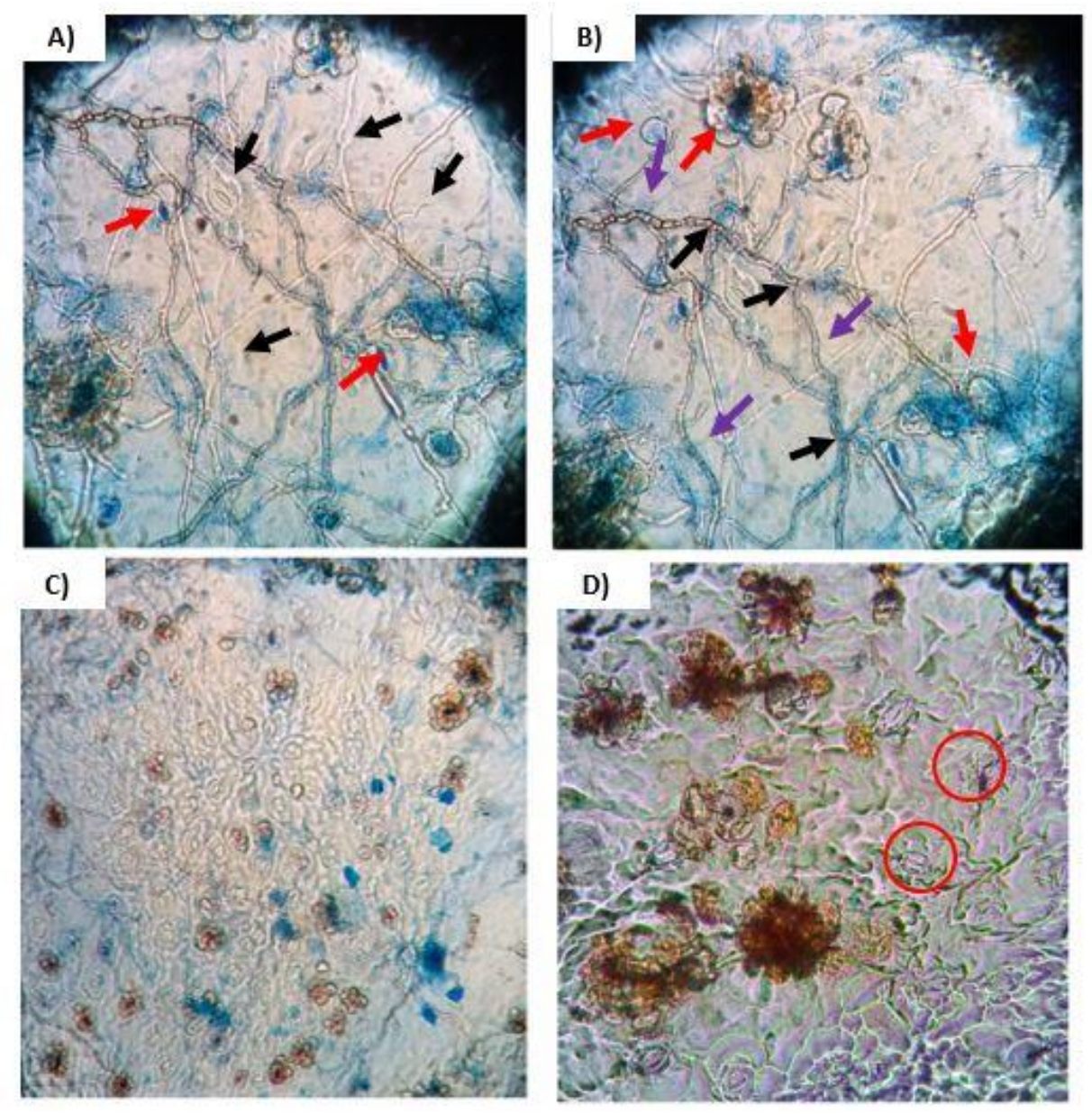

Fig. 1. Observación de cortes longitudinales del tejido foliar de plantas infectadas con H. vastatrix. A. Estomas de la hoja (flecha negra) y esporas dentro de estomas (flecha roja). B. Arreglo/aglomerado de urediniosporas (flecha roja), hifas septadas de germinación (flecha morada) e hifas de germinación unidas por diferentes urediniosporas (flecha negra). C. Vista del tejido foliar infectado. D. Vista del tejido foliar y estomas asimétricas (círculos rojos).

Con respecto a fenoles totales (FT), no hubo diferencias $(p<0.05)$ en las hojas de plantas Ni e i antes de la infección (ai) y durante ésta (di); sin embargo, durante el periodo de infección (di), la concentración de FT se incrementó al doble en la infectadas (i) (cuadro I).

En cuanto a los metabolitos secundarios, solo se observaron diferencias significativas en el contenido de cafeína y ácido gálico en hojas de plantas infectadas con roya. Sin embargo, antes de la infección el 5-CQA, 4-CQA y el ácido gálico no están presentes en las Ni; cabe señalar que las concentraciones de ácido gálico disminuyen en las i durante la infección, pero sigue siendo mayor que en las Ni. También se observó un claro incremento de cafeína en las hojas de i durante la infección (cuadro I).

La actividad de la quitinasa fue mayor en las hojas de plantas Ni, y está actividad se incrementó durante la infección (di). Por otro lado, la actividad de la glucanasa fue mayor en las i antes y durante la infección (ai y di, respectivamente). En cuanto a la actividad de la peroxidasa, ésta fue mayor en las Ni solo antes de la infección (ai) y esta actividad no varió durante la infección (di) en ambas muestras (Cuadro II). 
Cuadro I. Concentración de compuestos fenólicos en las hojas de C. arabica antes y durante la infección por roya.

\begin{tabular}{|c|c|c|c|c|c|c|c|c|c|}
\hline & & $\begin{array}{c}\text { 5- } \\
\mathrm{CQA}^{1}\end{array}$ & $\begin{array}{c}\text { 3- } \\
\mathrm{CQA}^{2}\end{array}$ & $\begin{array}{c}\text { 4- } \\
\mathrm{CQA}^{3}\end{array}$ & Ác. Cafeico & $\begin{array}{c}\text { Ác. } \\
\text { Ferúlico }\end{array}$ & Cafeína & $\begin{array}{c}\text { Ác. } \\
\text { Gálico }\end{array}$ & $\begin{array}{c}\text { Fenoles totales } \\
\text { mg Eq AG }\end{array}$ \\
\hline $\begin{array}{l}\text { Durante de la } \\
\text { infección }\end{array}$ & $\begin{array}{c}\text { Infectadas (i) } \\
\text { No infectadas (Ni) }\end{array}$ & $\begin{array}{l}6.3 b^{5} \\
33.5 \mathrm{a}\end{array}$ & $\begin{array}{l}507.9 \mathrm{a} \\
647.9 \mathrm{a}\end{array}$ & $\begin{array}{l}74.0 \mathrm{a} \\
69.5 \mathrm{a}\end{array}$ & $\begin{array}{l}\boldsymbol{\mu} \mathbf{g} \mathbf{E q} \\
12.2 \mathrm{a} \\
16.3 \mathrm{a}\end{array}$ & $\begin{array}{l}5.1 \mathrm{a} \\
4.2 \mathrm{a}\end{array}$ & $\begin{array}{l}5235.5 \mathrm{a} \\
4105.0 \mathrm{~b}\end{array}$ & $\begin{array}{l}5.3 \mathrm{a} \\
2.0 \mathrm{~b}\end{array}$ & $\begin{array}{l}6.0 \mathrm{a} \\
2.8 \mathrm{~b}\end{array}$ \\
\hline $\begin{array}{l}\text { Antes de la } \\
\text { infección }\end{array}$ & $\begin{array}{c}\text { Infectadas (i) } \\
\text { No infectadas (Ni) }\end{array}$ & $\begin{array}{c}39.8 \mathrm{a} \\
0 \mathrm{~b}\end{array}$ & $\begin{array}{l}695.6 \mathrm{a} \\
487.2 \mathrm{a}\end{array}$ & $\begin{array}{c}143.5 \mathrm{a} \\
0 \mathrm{~b}\end{array}$ & $\begin{array}{l}17.2 \mathrm{a} \\
11.3 \mathrm{a}\end{array}$ & $\begin{array}{l}2.1 \mathrm{~b} \\
7.2 \mathrm{a}\end{array}$ & $\begin{array}{l}351.7 \mathrm{~b} \\
8988.8 \mathrm{a}\end{array}$ & $\begin{array}{l}7.3 \mathrm{a} \\
0 \mathrm{~b}\end{array}$ & $\begin{array}{l}4.5 \mathrm{a} \\
4.4 \mathrm{a}\end{array}$ \\
\hline DMS $(0.05)^{4}$ & & 20.6 & 298 & 29.9 & 6.2 & 2.1 & 1074 & 3.1 & 0.965 \\
\hline $\begin{array}{c}\text { Interacciones } \\
\text { i x ai } \\
\text { i x di } \\
\mathrm{Ni} \times \text { di } \\
\mathrm{Ni} \text { x di }\end{array}$ & & $\begin{array}{l}496.5 \\
519.2 \\
894.6 \\
455.2\end{array}$ & $\begin{array}{l}496.5 \\
519.2 \\
894.6 \\
455.2\end{array}$ & $\begin{array}{c}148.0 \\
0 \\
139.0 \\
0\end{array}$ & $\begin{array}{c}11.4 \\
13.0 \\
23.0 \\
9.5\end{array}$ & $\begin{array}{l}2.3 \\
7.8 \\
1.8 \\
6.6\end{array}$ & $\begin{array}{c}362 \\
10109 \\
341 \\
7869\end{array}$ & $\begin{array}{c}10.6 \\
0 \\
4.0 \\
0\end{array}$ & $\begin{array}{l}5.5 \\
6.5 \\
3.4 \\
2.3\end{array}$ \\
\hline
\end{tabular}

Cuadro II. Actividades enzimáticas en las hojas de plantas de C. arabica antes y durante la infección por la roya.

\begin{tabular}{|c|c|c|c|c|}
\hline & & \multicolumn{3}{|c|}{ Actividad enzimática ${ }^{4}$} \\
\hline & & Quitinasa & Glucanasa & Peroxidasa \\
\hline & & $-\mu M$ p-nitrofenilo- & $-\mathbf{U A T}^{3}-$ & — mM purpurogalina- \\
\hline \multicolumn{5}{|r|}{ - mivi purpuroganina- } \\
\hline \multirow{2}{*}{$\begin{array}{l}\text { Antes de la } \\
\text { infección }\end{array}$} & Infectadas (i) & $0.113 \mathrm{~b}$ & $39.13 \mathrm{a}$ & $3.63 \mathrm{~b}$ \\
\hline & $\begin{array}{c}\text { No infectadas } \\
\text { (Ni) }\end{array}$ & $0.128 \mathrm{a}$ & $27.05 \mathrm{~b}$ & $6.09 \mathrm{a}$ \\
\hline \multirow{2}{*}{$\begin{array}{l}\text { Durante de la } \\
\text { infección }\end{array}$} & Infectadas (i) & $0.098 \mathrm{~b}$ & $43.61 \mathrm{a}$ & $4.59 \mathrm{a}$ \\
\hline & $\begin{array}{c}\text { No infectadas } \\
\text { (Ni) }\end{array}$ & $0.143 \mathrm{a}$ & $22.57 \mathrm{~b}$ & $5.13 \mathrm{a}$ \\
\hline $\operatorname{DMS}(0.05)^{1}$ & & 0.014 & 5.49 & 1.55 \\
\hline \multicolumn{5}{|l|}{ Interacciones } \\
\hline i x ai & & 0.116 & 54.4 & 4.66 \\
\hline i x di & & 0.109 & 23.87 & 2.60 \\
\hline $\mathrm{Ni} \times \mathrm{di}$ & & 0.081 & 32.8 & 4.52 \\
\hline $\mathrm{Ni} \times \mathrm{di}$ & & 0.175 & 21.28 & 7.66 \\
\hline \multicolumn{5}{|c|}{$\begin{array}{l}{ }^{1} \text { Diferencia mínima significativa }(0.05) ;{ }^{2} \text { Los valores en las columnas seguidos con la misma letra no son } \\
\text { estadísticamente diferentes entre los tratamientos; }{ }^{3} \text { Unidades de actividad total; }{ }^{4} \text { Todos los valores son por min/ } \square \mathrm{g} \text { de } \\
\text { proteína. }\end{array}$} \\
\hline
\end{tabular}




\section{DISCUSIÓN}

Las observación de cortes longitudinales del tejido foliar de plantas i corresponden a las características morfológicas de las urediniosporas de H. vastatrix (Fernándes, Evans, \& Barreto, 2009).

Estudios citológicos y bioquímicos han demostrado que en plantas de café se activan algunos mecanismos de defensa cuando es atacado por $H$. vastatrix. Uno de estos mecanismos es la respuesta hipersensible (HR), activada por efectores patogénicos), que se asocia con la deposición de callosa, compuestos fenólicos (flavonoides y ácido clorogénico) y con la lignificación de la pared celular. Otros tipos de respuestas involucran enzimas como las lipoxigenasas y las peroxidasas que estimulan la vía de los fenilpropanoides. Las proteínas PR (proteínas relacionadas con patógenos), como la $\beta$-1,3-glucanasa y la quitinasa, también se encuentran asociadas a la resistencia de algunos cultivares de café a $H$. vastatrix raza II (Fernandez et al., 2012; Florez et al., 2017).

La alteración de la concentración de los compuestos fenólicos comúnmente se encuentra relacionado con los mecanismos de defensa al estrés biótico (Ávalos \& Pérez, 2009). En el presente estudio se encontró que la concentración de fenoles totales (FT), no vario antes del proceso de infección y aumentó en las plantas i en comparación con las plantas Ni. Estos resultados indican que las plantas que son susceptibles a la enfermedad están respondiendo al ataque del fitopatógeno y que no es la presencia del hongo la que los induce.

Sin embargo, nuestros resultados indican también que las plantas de café podrían estar resistiendo al ataque de la roya debido a la producción de metabolitos inducidos como respuesta al ataque del hongo, es decir por resistencia sistémica inducida (RSI). Específicamente, los metabolitos que podrían estar implicados en la RSA son 5-CQA, cafeína y ácido gálico y los que posiblemente participen en la RSI son ácido ferúlico y la cafeína. Estos dos últimos metabolitos fueron detectados en mayor concentración en las hojas de las plantas Ni antes de la infección. El 5-CQA puede estar involucrado en el efecto de la capacidad antioxidante ante el estrés ocasionado por la presencia de la infección de $H$. vastatrix en la temporada anterior. También podría estar relacionado con la producción de cutinasa en la roya y con la reducción de la producción de melanina fúngica. La cutinasa es una enzima que el hongo usa para degradar la cutina de las hojas y poder adherirse a ellas permitiendo infectar a la hoja (Rodrigues, Carré, Jham, Berhow \& Schurt, 2011). Otra explicación es que este metabolito este asociado al proceso de lignificación de la pared celular vegetal y con esto promover el refuerzo de la primera barrera ante un ataque patogénico, además su acumulación podría mantener un índice en la lignificación o actividad antioxidante ante la presencia de una infección por $H$. vastatrix, y podría estar siendo usando con el fin de retardar el proceso infeccioso (Ávalos \& Pérez, 2009). Con respecto a la cafeína, la importancia radica en que es un metabolito que tiene alta actividad antioxidativa y es una molécula precursora en la síntesis de ácidos clorogénicos, por lo que su aumento podría estar ligado con la concentración del 5-CQA (Brezová \& Slebodová, 2009). Por otro lado, las plantas podrían estar usando los ácidos clorogénicos en una respuesta temprana de inhibir el crecimiento micelial o la germinación de esporas mediante la permeabilización de la membrana (Martínez, Regente, Jacobi, del Río, \& de la Canal, 2017). La diferencia de la concentración de ácido gálico podría ser explicada porque el ácido participa en la biosíntesis de taninos hidrolizables que pueden desempeñar actividad antifúngica (Ávalos \& Pérez, 2009). Es interesante el comportamiento de la actividad enzimática de la quitinasa ya que podría estar participando tanto en la RSA como en la RSI. Esta actividad enzimática degrada la pared celular del hongo y/o las hifas generadas por la esporulación y como consecuencia protege a la planta de la colonización del hongo (Guerra et al., 2009).

De acuerdo con González et al. (2011), la actividad enzimática de la glucanasa es una de las más importantes en el fenómeno de la resistencia vegetal ya que interviene en la degradación de los glucanos de la pared celular fúngica. Así mismo, Honorato et al. (2015) demostraron que la 
actividad de la glucanasa en las plantas se eleva en los días en torno a la infección de la roya desde el día 2 hasta el día 40, caso contrario a lo que se demuestra con las plantas control que no están infectadas. Monteiro et al., (2016) mostraron que las plantas de café ante la incidencia de la roya en las primeras 24 horas, hay un incremento de la expresión de la enzima glucanasa. El decremento de la actividad de glucanasa en plantas muestreadas antes de la infección con respecto a las plantas infectadas muestreadas durante la infección puede reflejar por qué la planta no contrarresta a la roya. La glucanasa puede no ser la enzima principal que le confiere defensa contra la roya a las plantas que no son infectadas y probablemente estas plantas utilizan principalmente a la enzima quitinasa para degradar la pared celular fúngica, además de usarla como un sistema protector durante la incidencia de la roya a posteriores ataques.

La actividad de la peroxidasa fue mayor en las plantas no infectadas antes y durante el proceso de infección, por lo que posiblemente esta enzima esté participando para mejorar el sistema antioxidativo y posiblemente usado como un mecanismo previsor ante agentes oxidantes como los que puede ocasionar el estrés al tejido foliar (Honorato et al., 2015). Se ha demostrado que la actividad de la peroxidasa induce la biosíntesis de lignina y suberina en hojas (Herrero et al., 2013), esto explicaría por qué las plantas resistieron el ataque de la roya.

\section{CONCLUSIONES}

Las plantas de $C$. arabica no susceptibles y por lo tanto resistentes a la roya presentaron niveles altos de 5-CQA y ácido cafeico durante la infección, y ácido ferúlico antes de ésta; en cuanto a las actividades enzimáticas, la quitinasa y peroxidasa mostraron mayor actividad antes y durante la infección, lo que sugiere un posible mecanismo de resistencia mediante la síntesis de éstos metabolitos y la actividad de éstas enzimas.

\section{AGRADECIMIENTOS}

Al Tecnológico Nacional de México por el financiamiento al proyecto. Jiménez-Aguilar L.A. recibió una beca del CONACYT.

\section{LITERATURA CITADA}

Ávalos, G., \& Pérez, U. (2009). Metabolismo secundario de plantas. Reduca (Biología). Serie Fisiología Vegetal, 2(3), 119-145.

Brezová, V., \& Slebodová, A. (2009). A coffee as a source of antioxidants: an EPR study. Food Chemistry, 114(3), 859-868. doi:10.1016/j.foodchem.2008.10.025.

Castillo, F., Hernández, D., Gallegos, G., Rodríguez, R. \& Aguilar, C.N. (2012). Antifungal properties of bioactive compounds from plants, fungicides for plant and animal diseases, Dr. Dharumadurai Dhanasekaran (Ed.), ISBN: 978-953-307-804-5, InTech, Available from: http://www.intechopen.com/books/fungicides-for-plant-and-animaldiseases/antifungal-properties-of-bioactivecompounds-from-plants

Chance, B., \& Maehley, A. (1955). Assay of catalases and peroxidases. Methods Enzymol, 2, 764-775.

Diniz, I., Azinheira, H., Figueiredo, A., Gichuru, E., Oliveira, H., Guerra-Guimaraes, L. \& Silva, M.C. (2019). Fungal penetration associated with recognition, signaling and defence-related genes and peroxidase activity during the resistance response of coffee to Colletotrichum kahawae. Physiological and Molecular Plant Pathology, 105, 119127. doi:10.1016/j.pmpp.2017.12.005.

Cuesta-Parra, D.M. \& Correa-Mahecha, F. (2018). Extration phenols from green coffee grains. Revista Ion, 31(1):31-35. 
Dogra, V., \& Sreenivasulu, Y. (2015). Cloning and functional characterization of $\beta-1,3-$ glucanase gene from Podophyllum hexandrum - A high altitude himalayan plant. Gene, 554(1), 25-31. doi:10.1016/j.gene.2014.10.012

Fernándes, R.D., Evans, H., \& Barreto, R. (2009). Confirmation of the occurrence of teliospores of Hemileia vastatrix in Brazil with observations on their mode of germination. Tropical Plant Pathology, 2, 108-113.

Fernandez, D., Tisserant, E., Talhinhas, P., Azinheira, H., Vieira, A.N.A., Petitot, A., \& Duplessis, S. (2012). 454-pyrosequencing of Coffea arabica leaves infected by the rust fungus Hemileia vastatrix reveals in planta -expressed pathogen-secreted proteins and plant functions in a late compatible plant - rust interaction. Molecular Plant Pathology, 13, 17-37. doi:10.1111/J.1364-3703.2011.00723.X.

FERTILAB. (2012). Manual de mustreo de suelo, planta y agua ( $3^{a}$ Edición ed.) Celaya.

Florez, J.C., Souto-Mofatto, L., do Livramento Freitas-Lopes R., Siqueira-Ferreira, S., MacielZambolim E., Falsarella-Carazzolle, M., Zambolim, L., \& Teixeira-Caixeta, E. (2017). High throughput transcriptome analysis of coffee reveals prehaustorial resistance in response to Hemileia vastatrix infection. Plant Molecular Biology. doi 10.1007/s11103-017-0676-7.

Guerra, G., Silva, M., Struck, C., Loureiro, A., Nicole, M., Rodrigues, C., \& Ricardo, C. (2009). Chitinases of Coffea arabica genotypes resistant to orange rust Hemileia vastatrix. Biologia Plantarum, 53(4), 702-706.

González, I., Infante, D., Peteira, B., Martínez, B., Arias, Y., González, N. \& Miranda, I. (2011). Biochemical characterization of promising isolates of Trichoderma spp. as biological control agents. II. Expression of glucanase activity. Revista de Protección Vegetal, 26, 23-29.

Haddad, F., Saraiva, R., Mizubuti, E., Romeiro, R., \& Maffia, L. (2013). Antifungal compounds as a mechanism to control Hemileia vastatrix by antagonistic bacteria. Tropical Plant Pathology, 38(5), 398-405.

Herrero, J., Fernández-Pérez, F., Yebra, T., Novo-Uzal, E., Pomar, F., Pedreño, M., Cuello, J., Guéra, A., Esteban-Carrasco, A. \& Zapata, J.M. (2013). Bioinformatic and functional characterization of the basic peroxidase 72 from Arabidopsis thaliana involved in lignin biosynthesis. Planta. 237, 1599-1612.

Honorato, J., Zambolim, L., Aucique, P., Resende, R., \& Rodrigues, F. (2015). Photosynthetic and antioxidative alterations in coffee leaves caused by epoxiconazole and pyraclostrobin sprays and Hemileia vastatrix infection. Pesticide Biochemistry and Physiology, 123, 31-39. doi:10.1016/j.pestbp.2015.01.016.

Loureiro, A., Gil, H., Maria, C., Talhinhas, P., \& Money, N.P. (2015). A method for obtaining RNA from Hemileia vastatrix appressoria produced in planta, suitable for transcriptomic analyses. Fungal biology, 119, 1093-1099. doi:10.1016/j.funbio.2015.08.008.

Martínez, G., Regente, M., Jacobi, S., del Río, M., \& de la Canal, L. (2017). Chlorogenic acid is a fungicide active against phytopathogenic fungi. Pesticide Biochemistry and Physiology 140, 30-35.

Monteiro, A. C. A., de Resende, M. L. V., Valente, T. C. T., Ribeiro Junior, P. M., Pereira, V. F., da Costa, J. R., \& da Silva, J. A. G. (2016). Manganese phosphite in coffee defence against Hemileia vastatrix, the coffee rust fungus: Biochemical and Molecular Analyses. Journal of Phytopathology, 164, 1043-1053.

Ramada, M. H. S., Lopes, F. Á. C., Ulhoa, C. J., \& Silva, R. do N. (2010). Optimized microplate $\beta$-1,3-glucanase assay system for Trichoderma spp. screening. Journal of Microbiological Methods, 81(1), 6-10. doi:10.1016/j.mimet.2010.01.010

Rodrigues, F., Carré, M., Jham, G., Berhow, M., \& Schurt, D. (2011). Chlorogenic acid levels in leaves of coffee plants supplied with silicon and infected by Hemileia vastatrix. Tropical Plant Pathology, 36(6), 404-408.

Silva, M., Nicole, M., Rijo, L., Geiger, J., \& Rodrigues, C. (1999). Cytochemistry of plant-rust fungus interface during the compatible interaction Coffea arabica (cv. Caturra)Hemileia vastatrix (race III). International Journal of Plant Sciences, 79-91. 


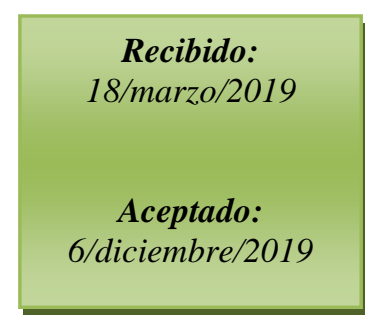

Temis, P., Lópes, M., \& Sosa, M. (2011). Producción de café (Coffea arabica L): cultivo, beneficio, plagas y enfermedades. Temas Selectos de Ingeniería de Alimentos, 5(2), 54-74.

Vignoli, J.A., Bassoli, D.G. \& Benassi, M.T. (2011). Antioxidant activity, polyphenols, caffeine and melanoidins in soluble coffee: The influence of processing conditions and raw material. Food Chemistry, 124, 863-868. 